Original Research Paper

\title{
Genomic Signature of Respiratory Microbes on Human Scalp Epithelia
}

\author{
${ }^{1}$ Bhola Nath Paul, ${ }^{2}$ Vani Mishra, ${ }^{1}$ Jasmeet Kaur Chhabra and ${ }^{3}$ Divyani Paul \\ ${ }^{I}$ Immunobiolgy Division, CSIR-Indian Institute of Toxicology Research, Lucknow, India \\ ${ }^{2}$ Nanotechnology Application Centre, Allahabad University, Allahabad, India \\ ${ }^{3}$ Biophysics Division, All India Institute of Medical Sciences, New Delhi, India
}

Article history

Received: 18-12-2014

Revised: 13-01-2015

Accepted: 17-04-2015

Corresponding Author: Bhola Nath Paul Immunobiolgy Division, CSIRIndian Institute of Toxicology Research, Lucknow, India Email:paulbnath@gmail.com

\begin{abstract}
Despite overlap between habitats occupied by diverse Airborne Respiratory Microbes (ARM), the airway epithelium is known for respiratory microbe infections, human scalp epithelium is undocumented. Analogous to airways epithelium, the scalp epithelium is vulnerable to infection by dormant variants of sensitive microbes that underlie many chronic and relapsing diseases, difficult to eradicate by conventional antibiotics and challenged our ability to treat chronic infection. Hence comprehensive understanding of scalp epithelium ARM load, its disposal and impact on the host, as well as the environment, remains to be defined; we need to know the portals of entry and the virulence potential of ARM in determining the impact on human health and well beings. Here we investigate the genomic signature of diverse ARM in inflammatory scalp programmed for desquamation. Spatial analysis of Mycobacterium tuberculosis (Mtb), Chlamydophila pnumoniae (Cp), Escherichia coli (E. coli), Respiratory Syncytial virus strain A/B (RSVA/RSVB) and Influenza virus (H1N1) in the Flakes of White Scales (FWS) from the hair bearing areas of the scalp by quantitative (q) PCR reveals flux of microbes ranging from $\sim 10^{6}$ to $10^{8}$ copies/ng FWS-DNA. Mtb and 16SrRNA identity reaffirmed by amplicon sequencing. Absence of microbe gene expression signatures in FWS negates the presence of viable ARM, absence of $E$. coli adhesin mRNA indicates habitat rejection in coculture of epithelial cells and E. coli JM107 and absence of H1N1 hemaglutinin1 (H1) mRNA in FWS rules out canonical binding and downstream infection of neighboring cell by $\mathrm{H} 1 \mathrm{~N} 1$. We conclude that desquamated epithelium, in addition to Malassezia sp, bear diverse genomic signatures of non-virulent respiratory microbes, suggest an undefined portal of entry, helps in clearance of microbes and possess minimum risk of re-infection to neighboring cells and subjects.
\end{abstract}

Keywords: Respiratory Microbes Epithelium Human Infection

\section{Introduction}

Bacteria and viruses are ubiquitous in the atmosphere, with concentrations of bacterial cells typically exceeding $1 \times 10^{4} \mathrm{~m}^{-3}$ over land (Burrows et al., 2009), while airborne viral abundance exhibited a seasonal fluctuation in the range between $1.7 \times 10^{6}$ to $4.0 \times 10^{7} \mathrm{~m}^{-3}$ (Whon et al., 2012). These microbes tend to establish an infectious foothold on the human epithelium; in defense, the epithelium countermeasures against intruders through multiple layers of microbial sensing and intrinsic defense systems (Kim et al., 2010). Flakes of White Scales (FWS) from the hair bearing areas of the scalp are primarily considered for association with Malassezia infection (Saunders et al., 2012); the phylogenetically distant respiratory microbes like Mycobacterium tuberculosis $(M t b)$, Chlamydophila pnumoniae $(C p)$, Respiratory Syncytial virus strain $A / B$ (RSVA/RSVB) and Influenza virus (H1N1) are commonly unrepresented. Mtb is the etiological agent of Tuberculosis (TB) in humans that has plagued human populations for thousands of years, 9 million people fell ill to TB in 2013 , killed 1.5 million 
people and 480,000 developed Multiple Drug Resistant TB (MDR-TB) globally according to the World Health Organization (WHO, 2014). Cp, a common cause of human respiratory disease, is an intracellular pathogen responsible for a number of different acute and chronic infections, including cardiovascular (atherosclerosis and stroke) and Central Nervous System (CNS) disorders (Contini et al., 2010). H1N1 virus is the subtype of influenza A virus, the most common cause of human influenza (flu) and responsible for the 2009 pandemic. Incidentally, it predominated overall during the 2013-14 flu season in USA. Nearly $60 \%$ of the fluassociated hospitalizations reported to CDC's influenza surveillance system were in people 18 to 64 years old (CDC, 2014a). $R S V$ is also a respiratory virus that infects the lung and breathing passages. Though healthy people recover from $R S V$ infection in 1-2 weeks, infection can be severe in certain infants, young children and older adults. It is the common cause of bronchiolitis and pneumonia (CDC, 2014b). E. coli is a non-respiratory microbe having various modes of transmission, including through food and water sources, animal-to-human contact and personto-person contact in different settings. One of the common modes of transmission occurs through fecal-oral route and cause diarrhea and urinary tract infection (Croxen et al., 2013). Airborne transmission of microbes occurs by dissemination of either airborne droplet nuclei or small particles in the respirable size range containing infectious agents that remain infective over time and distance. The impact of biological aerosol exposure on human health and well being are considerable. These ARM are a major cause of respiratory ailments of humans, causing allergies, asthma and pathogenic infections of the respiratory tract. Removal of infected cells is necessary for the maintenance of overall health and homeostasis and prevention of autoimmunity, pathogen burden, or cancer (Hochreiter-Hufford and Ravichandran, 2013). The neutrophils play a critical role in the swift clearance of infection. Rapid recruitment of neutrophils at the site of infection (Miller, 2013), engulfment of microbes followed by apoptosis and the occurrence of leukocyte extravasations mainly in the post-capillary venules where haemodynamic shear forces are minimum (Weninger et al., 2014), may help promote clearance of infection. Here we measure the composition and load of few respiratory microbes in FWS, as well as the virulence potential of desquamated human scalp cells. This study elaborates the presence of genomic signatures of diverse respiratory microbes in desquamated epithelium, defines an aberrant portal of entry and associated health hazard(s) in human.

\section{Materials and Methods}

\section{Study Design}

FWS from the laundry coats were collected during the winter months of the year 2012 and 2013 from local Dry
Cleaners, weighed and washed thrice with Phosphate Buffered Saline (PBS) containing non-ionic detergent, Tween-20 (0.01\%) to remove the passively bound microbes. Samples (containing $>20$ flakes) were considered for the study. Samples were collected with the help of plastic forceps and split into $\sim 2$ equal fractions. One fraction was incubated with proteinase $\mathrm{K}$ for $5 \mathrm{~min}$ and thereafter with Buffer $\mathrm{AL}$ for $10 \mathrm{~min}$ at $56^{\circ} \mathrm{C}$ and DNA eluted in water using DN easy blood and tissue kit (Qiagen). Purified DNA has $\mathrm{A}_{260} / \mathrm{A}_{280}$ ratios of 1.7-1.9 and the absorbance scans shows a symmetric peak at $260 \mathrm{~nm}$ confirming high purity. These DNA were used as templates for standard PCR (HotStarTaq ${ }^{\circledR}$ Master Mix kit, Qiagen) and Real-time PCR (Quantitect SYBR ${ }^{\circledR}$ PCR kit) to study the pattern of microbial distribution and copy numbers of microorganism, respectively. Other fractions of the samples were subjected to total RNA isolation based on combined selective binding properties of a silica based membrane with the speed of microspin technology (RNeasy Mini Kit, Qiagen). Purified RNA was used for quantification of microbial mRNA by using one step RT-PCR kit (Qiagen). Since the results were negative (Supplementary Fig. 1), amplicon sequencing were not pursued. HIV- and hepatitis B- and C-negative blood from healthy volunteers was procured from a local Blood Bank in Lucknow, India for the isolation of Granulocytes following the method described elsewhere (Chhabra et al., 2014).

\section{Isolation of Oral Epithelial Cells}

The epithelial cells were prepared by gentle scraping of the soft palate at the back of the mouth roof (Donor: BNP). Tissue containing oral epithelium were transferred in Dulbecco's Modified Eagle's Medium supplemented with $10 \%$ fetal bovine serum, penicillin $\left(100 \mu \mathrm{g} \mathrm{mL}^{-1}\right)$, streptomycin $\left(100 \mu \mathrm{g} \mathrm{mL}^{-1}\right)$ and gentamycin $\left(50 \mu \mathrm{g} \mathrm{mL}^{-1}\right)$ solutions and incubated at $37^{\circ} \mathrm{C}$ for $2 \mathrm{~h}$ for sterilization. Tissues were then washed in PBS without $\mathrm{Ca}^{++}$and $\mathrm{Mg}^{++}$several times before initiation of coculture experiments E. coli JM107. Viability checked by trypan blue dye exclusion was $>97 \%$.

\section{Culture of E. coli JM107}

The E. coli strain JM107, (Fermentas Life Sciences, Burlington, Canada), is a non-pathogenic bacterium derived from the $E$. coli $\mathrm{K} 12$ strain lacking the $\mathrm{omp}^{\mathrm{T}}$ gene (Wu et al., 2003). It has a mean generation time of $25 \mathrm{~min}$ and a lag period of $70 \mathrm{~min}$. Bacteria were subcultured and maintained on LB agar plates. Colonies were scraped, washed, resuspended in LB media and grown at $37^{\circ} \mathrm{C}$ and $115 \mathrm{rpm}$ in a shaker incubator until the late logarithmic phase. The bacteria were harvested by centrifugation at $4000 \times \mathrm{g}$ for $5 \mathrm{~min}$, washed, resuspended in Ringer's solution and quantified spectrophotometrically $\left(1 \mathrm{OD}_{600}=10^{9}\right.$ bacteria $\left./ \mathrm{mL}\right)$. 


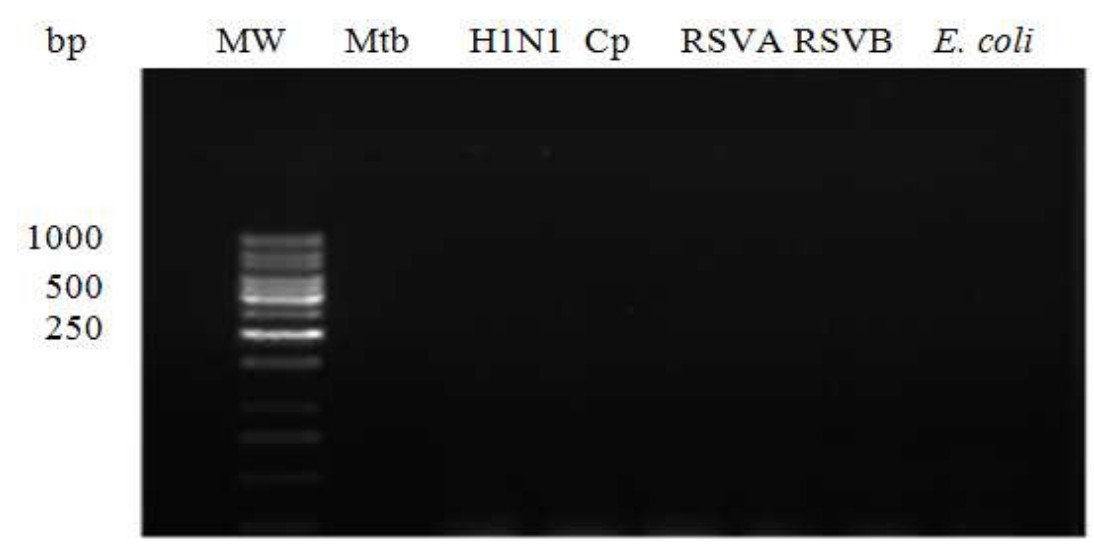

Supplementary Fig. 1. Representative mRNA profiles of different microbes in FWS

\section{Coculture of Epithelial Cells and JM107}

In vitro co-culture of epithelial cells and E. coli $J M 107$ were performed to optimize the concentration of Tween-20 for removal of passively adhering bacteria on epithelial cells. We cocultured unviable epithelial cells $\left(10^{4}\right)$ and 10 fold excess E. coli JM107 $\left(10^{5}\right)$ in triplicate wells for $180 \mathrm{~min}$. After washing (3X) with varying concentration of Tween-20, DNA was isolated and used as template for PCR amplification with E. coli primers. Cells from one of the well was stained with Acridine Orange (AO) dye for fluorescence microscopy. Unviable epithelial cells were prepared by treating epithelial cells with $50 \%$ ethanol in 2X Phosphate Buffered Saline (PBS) for 1 h. Experiments were performed on unviable cells to mimic FWS. Experiments were also performed with viable epithelial cells in a similar way for microscopic observation. Ethanol pretreatment was avoided for experiments with viable epithelial cells.

\section{Evaluation of Hemagglutinin1 (H1) and Adhesin} $m R N A$

$\mathrm{H} 1$ and adhesin expression profiles were measured by Reverse Transcriptase (RT)-PCR. H1 mRNA levels were measured from the total RNA isolated from influenza virus positive FWS $\left(\mathrm{FWS}^{\mathrm{H} 1 \mathrm{~N} 1+}\right)$, negative FWS $\left(\mathrm{FWS}^{\mathrm{HIN1}-}\right.$ ) and H1N1 Mexican lysates (Genome Diagnostics, New Delhi) by using one step RT-PCR kit (Qiagen). E. coli adhesin was measured in coculture of epithelial cells and JM107 in presence/absence of TNF- $\alpha$ pretreated granulocytes and other settings.

\section{Primer Sequences}

C. pnumoniae, F:5'TGACAACTGTAGAAATACAGC3' \& R: 5'ATT TATAGGAGAGAGGCG 3' E. coli $\mathrm{F}$ : 5'GGAAGTTT TCAGAGATG AGAA $3^{\prime}$ \& R:5'CCTTCGAGGAG GGCG C TT 3'; Human $\alpha$-globin, F:5'GGCAGACTTCTCCTCA GGA GTC3' \&
R:5'CTTAGACCTCA CCCCTGTGGAGC3'; $M$. globusa, F:5'AACTTAAAGGAA TTGACGGAAG3' \& R: 5' GGCAGGAACCAGCTACTAG; M. tuberculosis F:5'CACA TGCAAGTCGAACGGAAAGG3' \& R: 5'GCCC GTATCG CCCGCACGCTCACA 3'; RSVA, F:5'GATGTTACGGTG GGGAGTCT3' \& R:5'GTACACTGTAGTTAATCA CA 3'; $R S V B, \mathrm{~F}$ : 5'AATGCTAAGATGGGGAGTTC3' \& R: 5'GAA ATTGAGTTA ATGACAGC 3'; Swine flu (H1N1) F: 5' TGA TCTTCTTGAAAATT TGCA3' \& R:5'TGTTGACA AAAT GACCATCG3'; 16SrRNA, F:5' CCCAATTCGTCGAC AA

AGAGTTTGATCCTGGCTCAG3'\& R: 5'CCCGGGAT CC AAGCTTACGGTTACCTTGTTACGACTT3'; E. coli adhesin, F:5'GACGGCTGTACTGCAGGGTGTGGC3' \& $R$ : 5 'ATAT CCTTTWCTGCAGGGATGCAATA3' and Influenza H1, F:5'ATTGCCGGTTTCATTGAAGG3' \& R:5'ATGGCATT YTGTG TGCTYTT3'.

\section{Results}

\section{Dose Optimization of Tween-20}

Different species of fungi (Malassezia) are the ubiquitous skin residents of humans and other warmblooded animals that play a critical role in disorders including dandruff and seborrheic dermatitis and affect $>50 \%$ of humans ( $\mathrm{Xu}$ et al., 2007). E. coli is a non-respiratory and non-fungal microbe; we adopted this bacterium for optimizing the Tween-20 dose for the removal of passively bound microbes on FWS. The epithelial cells are washed with a non-ionic surfactant, tween-20 $\left(\mathrm{C}_{58} \mathrm{H}_{114} \mathrm{O}_{26}\right)$ (Fig. 1a) at non-cell lysing concentrations $(0-0.02 \%)$. The threshold concentration of water miscible Tween-20 that effectively removes passively bound microbes from the epithelial cells in comparison to PBS was found to be $0.01 \%$ (Fig. 1b-f). 
a

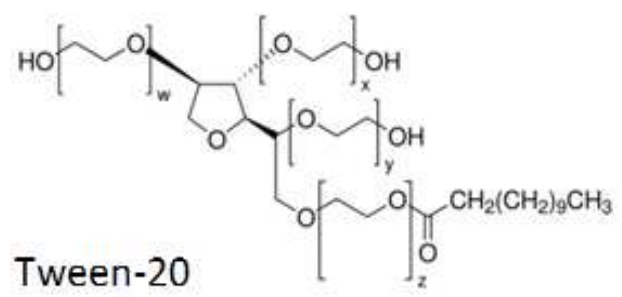

$\mathrm{b}$

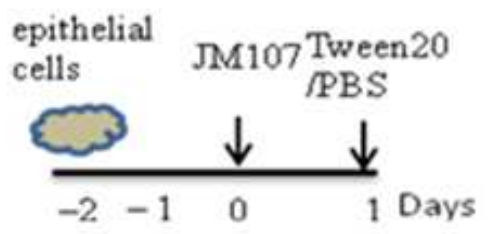

$\mathrm{c}$

d

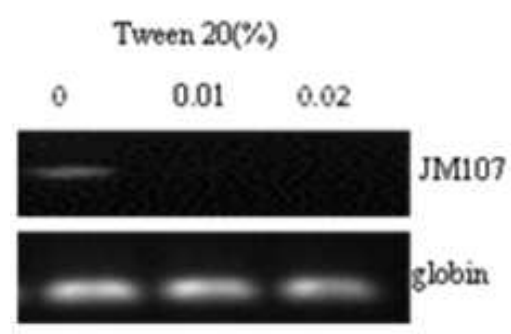

d

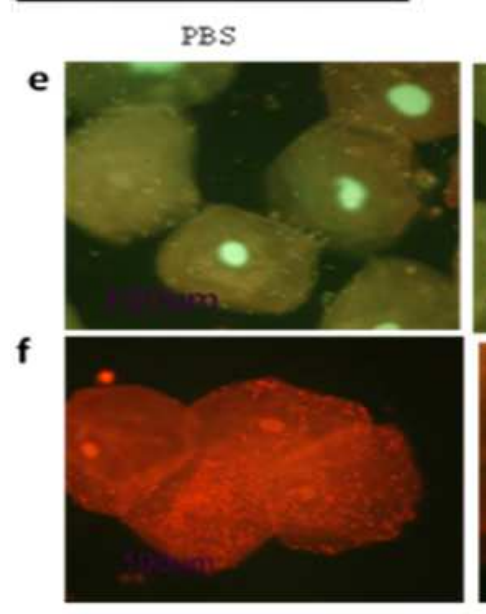

PBS+Tween20 (0.01\%)
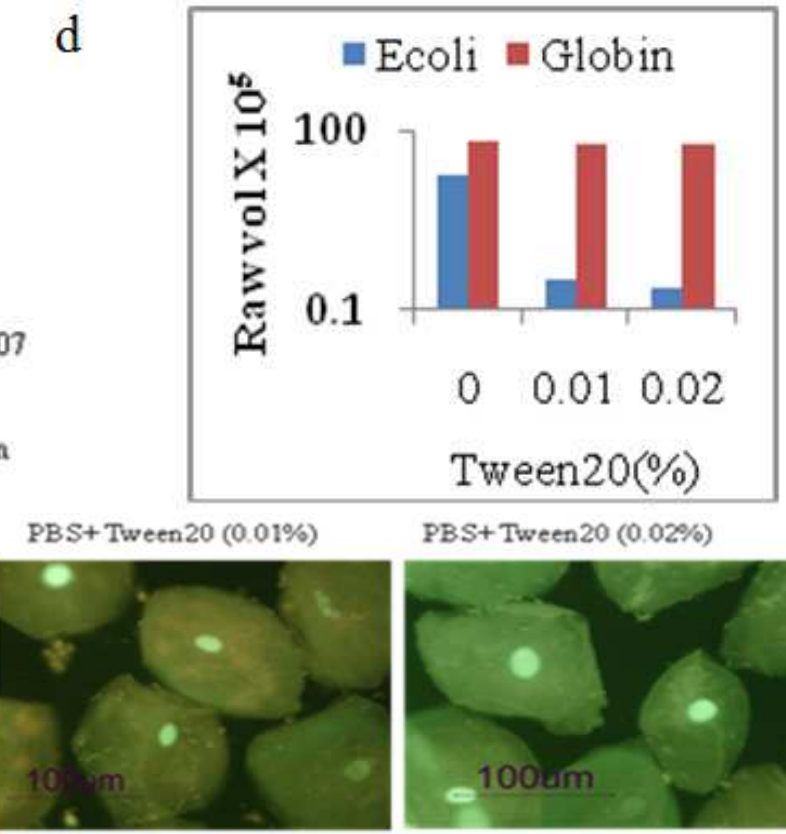

PBS+ Tween $20(0.02 \%)$
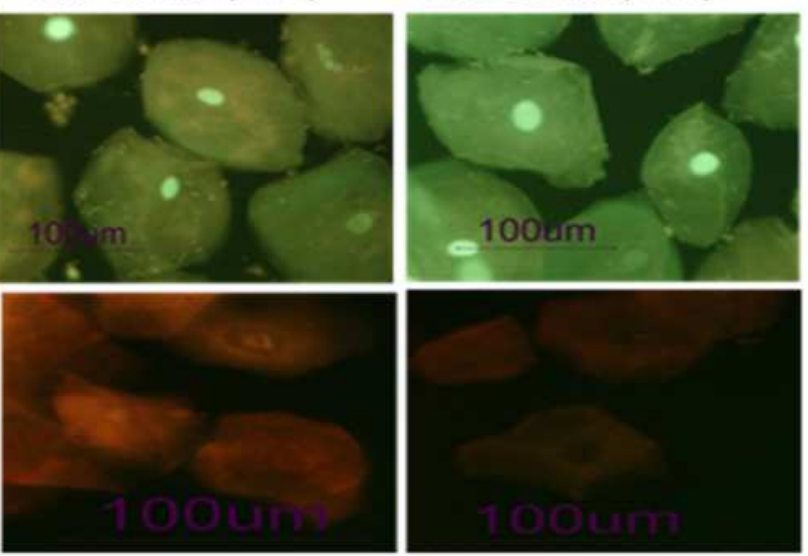

Fig. 1. Tween-20 restricts passive binding of E. coli JM107 on epithelial cells. (a) Chemical structure of Tween-20; (b) Protocol schema; (c) E. coli amplicons in dead epithelial cells cultured with JM107; after $24 \mathrm{~h}$, washed with varying doses of Tween-20, DNA isolated and PCR amplified; (d) densitometric score of c (e) AO stained images of Tween-20 washed viable epithelial cells cultured with JM107; (f) same as e except the epithelial cells are alcohol pretreated

\section{Microbial Composition and Load}

Occurrence of respiratory microbes in FWS is not yet documented, we demonstrate the presence of $M t b, C p$, RSVA, RSVB and H1N1, as well as $16 \operatorname{SrDNA}$ amplicons and non-respiratory $E$. coli in surfactant washed FWS samples (Fig. 2a) with variable probability (Fig. 2b). Human $\alpha$-globin (Mglo) serves as internal control. One third of the global population is currently infected with Mtb (Bartek et al., 2014), we report here a low distribution probability (0.009) of Mtb in FWS.
To determine whether the $M t b$ amplicons represent the TB bacillus, we sequenced a representative Mtb positive PCR product (Fig. 2c). It reveals $92 \%$ homology with $3 \%$ gaps in relation to the reference sequence, NC_000962.3 (Supplementary Fig. 2). The BLAST results between the query and the $\mathrm{NC}_{-} 000962.3$ sequences are presented as the NCBI dot matrix view (Fig. 2d) and the alignments are shown in the plot as lines; the number of lines shown in the plot is the same as the number of alignments found. The low gap score and high identity reflect a strong alignment, provides 
evidence for closely related sequences and establishes the presence of $M t b$ in FWS.

16SrDNA sequencing plays a pivotal role in the accurate identification of bacterial isolates and the discovery of novel bacteria in clinical microbiology laboratories (Kim et al., 2012), especially the Mycobacterium genera, despite its limited differentiation capability among Mycobacterium avium intracellulare, M. paratuberculosis, M. Chelonae and M. abscessus and members of the $M t b$-complex (Woo et al., 2008). The largest number of novel species discovered is of the genera Mycobacterium (Kim et al., 2012). Nucleotide sequencing of a representative 16SrDNA amplicons show $>90 \%$ homology with $3.5 \%$ gaps in relation to reference sequence in the Greengenes database
(Supplementary Fig. 3). Blast implementation searches for significant alignments between nucleotide sequence of 16SrDNA PCR-product from FWS (sample \#12) and 16SrRNA gene data base at Greengenes did not show identity with $M t b, C p$ and $E$. coli species, but reveals identity ranging between 76.87-71.41 with different strains like Staphylococcus capitis, Staphylococcus capre, Staphylococcus epidermidis (Table 1). Low identity rules out the presence Staphylococcus $s p$. in the exfoliating flakes. However, presence of H1N1, RSVA and $R S V B$ amplicons in FWS (Fig. 2a and $2 \mathrm{~b}$ ) suggests that the exfoliating flakes act as a depot of several respiratory viruses in addition to respiratory bacteria and fungus, Malassezia $s p$.
FWS

a $1 \begin{array}{llllllllllll}1 & 2 & 3 & 4 & 5 & 6 & 7 & 8 & 9 & 10 & 1112 & 13\end{array}$
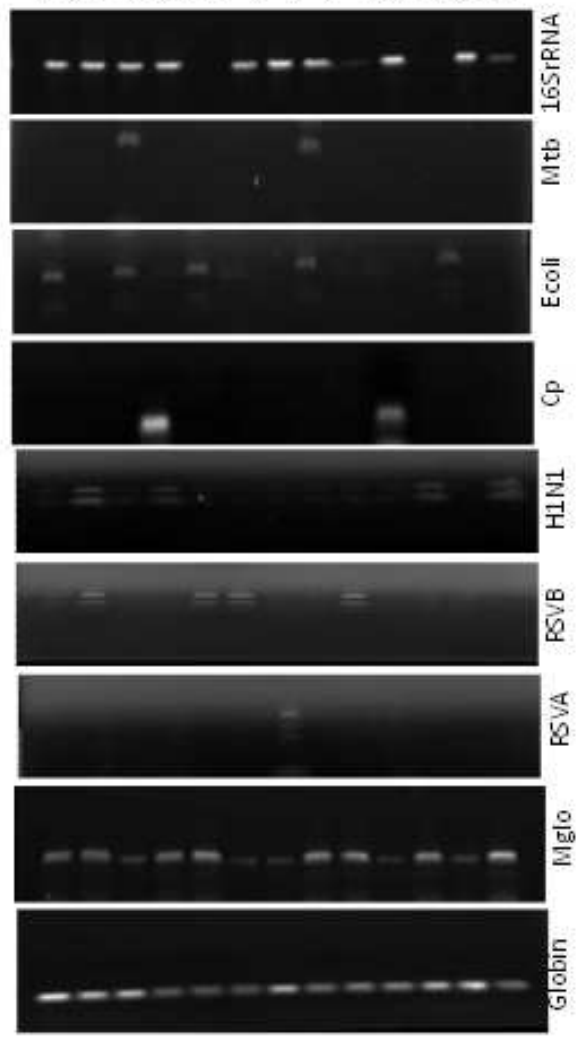

b

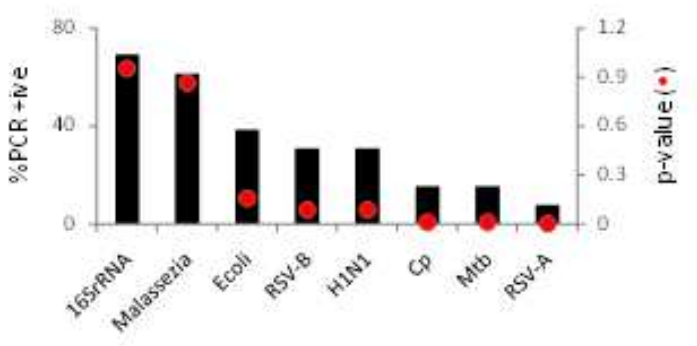

C

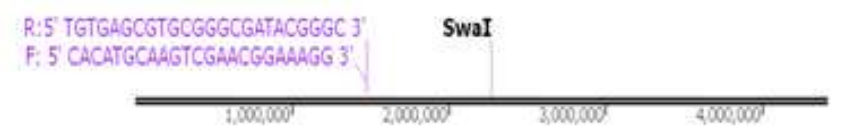

Mtb [1..4411532] Accession NC_000962.3

d

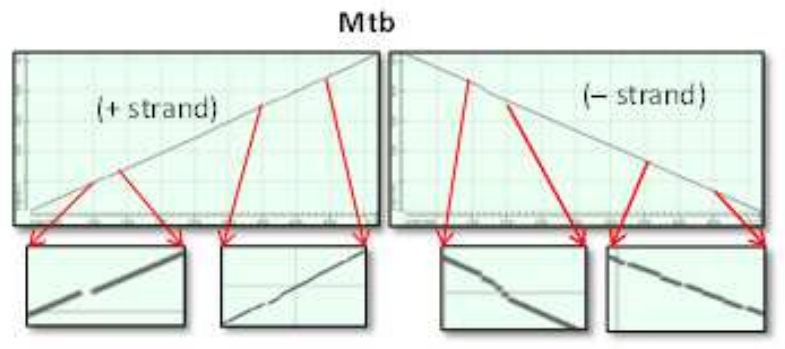

Fig. 2. Microbiome load and distribution in human FWS; (a) assessment of different microbial amplicons in FWS; (b) Rank plot showing distribution probability of microbes in FWS samples $(\mathrm{n}=13)$. P-values were derived from binomial distribution probability (Excel-BINODIST) and represented by red dots; (c) position of primer set on Mtb genome. (d) NCBI dot matrix view showing regions of similarity based upon the BLAST results between the Mtb sequence and the subject sequences. The PCR amplicon sequence (query sequence) is represented on the $\mathrm{X}$-axis and the numbers represent the bases/residues of the NCBI reference sequence, NC_000962.3, is represented on the Y-axis. Both PCR and RT-PCR are performed to detect H1N1 virus in FWS comprising of dead corneocytes. While H1N1 virus mRNA are undetectable by RT-PCR, standard PCR amplicons are visible in few FWS samples despite H1N1 being a RNA virus. The ability to detect $H 1 N 1$ cDNA in FWS arises preferably from the viral replication phase that requires a DNA intermediate in the host cell cytoplasm. FWS are likely to contain these DNA intermediates of H1N1 that act as template for standard PCR amplification. Like HIN1 Virus, RSVA and RSVB are negative-sense, single-stranded RNA virus of the family Paramyxoviridae. Presence PCR amplicons can be attributed to the mechanism discussed for H1N1 


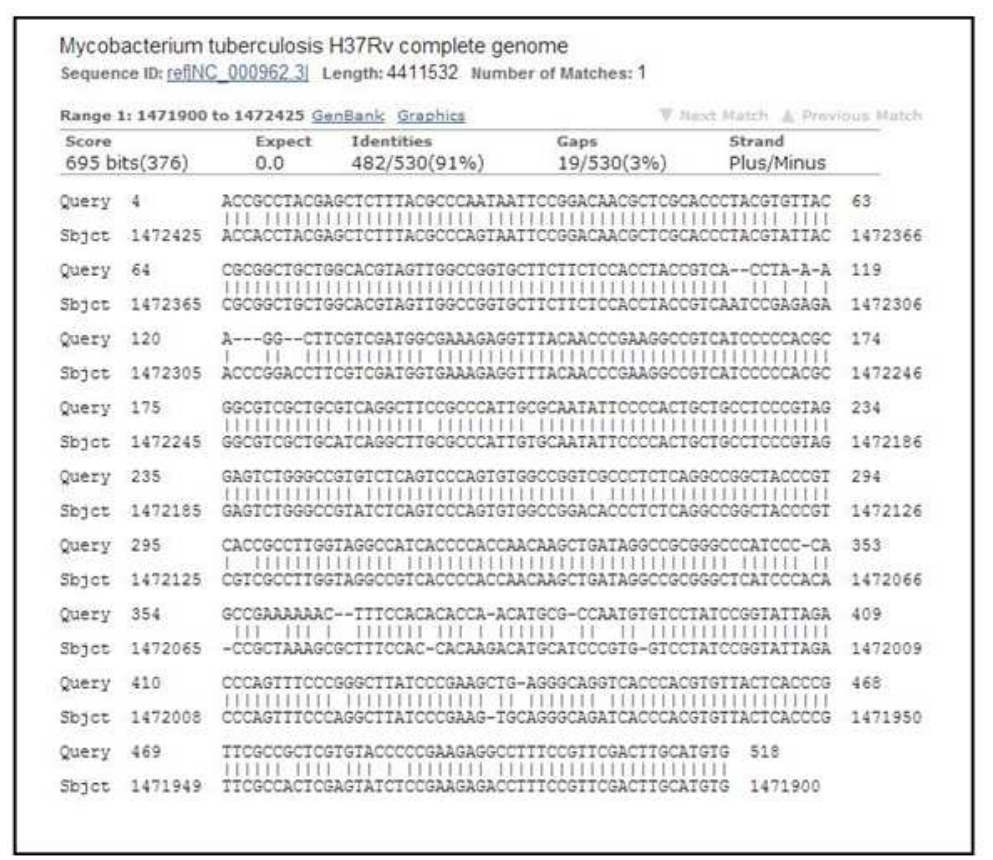

Supplementary Fig. 2. Blast result between sequences of Mtb amplicon and NCBI H37Rv

Table 1. Blast summary of 16SrDNA sequence with Greengenes database (http://greengenes.lbl.gov/cgi-bin/nph-last_interface.cgi)

\begin{tabular}{|c|c|c|c|c|}
\hline Staphylococcus species & Acc. \# & Score & Identity & Match length \\
\hline \multicolumn{5}{|l|}{ epidermidis str. } \\
\hline MVBDUS & HM636927.1 & 478 & 76.47 & 816 \\
\hline BQN1N-02d & FJ380964.1 & 413 & 71.41 & 801 \\
\hline MSFC3-MS-R1 & DQ447776.1 & 414 & 71.41 & 801 \\
\hline \multicolumn{5}{|l|}{ capitis str } \\
\hline BBN2R-02d & FJ357599.1 & 479 & 76.87 & 801 \\
\hline BQEN3-03 & FJ380955.1 & 479 & 76.87 & 801 \\
\hline BBN3T-04d & FJ357614.1 & 479 & 76.87 & 801 \\
\hline BBER-01d & FJ357585.1 & 479 & 76.87 & 817 \\
\hline BBN2P-01d & FJ357596.1 & 479 & 76.87 & 817 \\
\hline BBN3P-01d & FJ357608.1 & 479 & 76.87 & 817 \\
\hline BBEC-1d & FJ357580.1 & 479 & 76.87 & 817 \\
\hline BBN2R-02d & FJ357599.1 & 413 & 71.41 & 801 \\
\hline \multicolumn{5}{|l|}{ caprae str } \\
\hline BAC2039 & HМ355630.1 & 413 & 71.41 & 801 \\
\hline ATCC 35538 & NR_024665.1 & 413 & 71.41 & 801 \\
\hline \multicolumn{5}{|l|}{ sp.str. } \\
\hline SIIA16437 & FJ492836.1 & 413 & 71.41 & 801 \\
\hline SIIA 2055 & FJ492844.1 & 413 & 71.41 & 801 \\
\hline JDE-2009 80 & FN435890.1 & 413 & 71.41 & 791 \\
\hline
\end{tabular}

To quantify the different microbial load in FWS, we measured the copy number of $M t b, C p, E$. coli, H1N1, $R S V A, R S V B$ and 16SrRNA amplicons by absolute quantification using the standard curve method. Quantitative (q) PCR is increasingly used for the quantification and identification of microorganism in different disease states (Zemanick et al., 2010). The copy number of pathogens (/ng FWS-DNA) predominating in different FWS samples vary randomly and reveals $2.99 \times 10^{6}-1.91 \times 10^{7}$ copies of E. coli, $2.87 \times 10^{7}-6.38 \times 10^{8}$ copies of $H 1 N 1$ and $1.24 \times 10^{7}-1.9 \times 10^{8}$ copies $R S V B$ as shown in the area chart (Fig. 3a). Mtb, $C p$ and $R S V A$ are the rare pathogens in FWS and the copy number varies between $10^{6}-10^{8}$ in FWS. PCR amplicons are used as standards and the concentrations measured by $\mathrm{A}_{260}$ are converted to the number of copies using the molecular weight of the cDNA. The PCR efficiency ranged from 2.5 to 1.938 while deriving the standard curves for $16 \mathrm{SrRNA}$ (Fig. 3b) and $M t b$ (Fig. 3c). The slope of the standard curve is translated to an efficiency value by the Light Cycler ${ }^{\circledR} 480$ software. The mean squared error of the single data points fit to the regression line is represented 
by error value and lie within the acceptable limit $(<0.2)$. The standard curves for HIN1 and $R S V-B$ (Supplementary Fig. 4a) too reflected PCR efficiency within the practical limits and the mean squared error and are within the acceptable range.

Since the first negative derivative of the sample fluorescent curves results in a single peak for each positive $M t b$ (Fig. 3d), 16SrRNA (Fig. 3e) and H1N1, RSVB, $R S V A, E$. coli and $C p$ (Supplementary Fig. 4b), it proves amplification of the specific target sequence and no byproducts. Collectively, these data highlight the complex nature of FWS microbiome and polymicrobial infections are common with substantial non-viable microbe load. Although our knowledge of cell desquamation in response to respiratory microbe infection is unclear, the present study indicates that expulsion of non-viable infectionladen epithelial cells is an important intrinsic defense system against bacterial and viral colonization and confines pathogen spreading within the host, as well as the environment. Additionally, these data mirror the hostmicrobe association and sterility quotient of environment.

\section{Status of Adhesin and Hemaglutinin1 mRNA in FWS}

The presence of diverse respiratory microbes in FWS can result, either by direct precipitation and infection of the superficial scalp epidermis, stratum corneum, or through a process of migration from different loci. In vitro epithelial cell infection assays are performed to test for adaptation to the skin epithelial environment by coculturing human epithelial cells and JM107 in presence/absence of TNF- $\alpha$ primed granulocytes (Fig. 4a). DNA purified from Tween-20 washed epithelial cells previously cultured with JM107 in absence of granulocytes reveal the absence of $E$. coli amplicons, oppose to culture with TNF- $\alpha$ primed granulocytes (Fig. $4 \mathrm{~b}$ and $4 \mathrm{c})$. It suggests the inability of bacteria to adapt to epithelial cell ecology and granulocytes are prerequisite for displaying $E$. coli genomic signature in epithelial cells. Furthermore, adherence is an essential step in bacterial infection required for colonizing a new host and bacterial pathogens are able express an array of different adhesins (Clements et al., 2012). Inhibiting a single adhesin molecule can often make pathogenic bacteria non-virulent. Analysis of $E$. coli adhesin molecules in different experimental settings (Fig. 4a) reveal habitat rejection and obligate requirement of granulocytes (Fig. $4 b$ and $4 c$ ) for displaying genomic signatures in epithelial cells. Similarly, absence of Hemaglutinin1 (H1) mRNAs in $\mathrm{FWS}^{\mathrm{H} 1 \mathrm{N1+}}$ (Fig. 4d) rules out canonical binding and infection of H1N1 on anucleated keratinocytes of stratum corneum. H1 is the key molecule in binding and invasion in cells (Shelton et al., 2011).

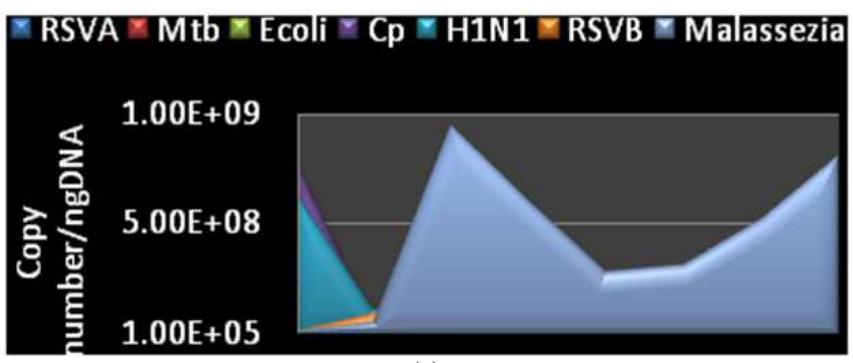

(a)

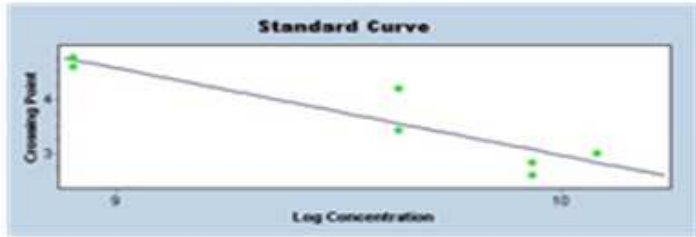

(b)

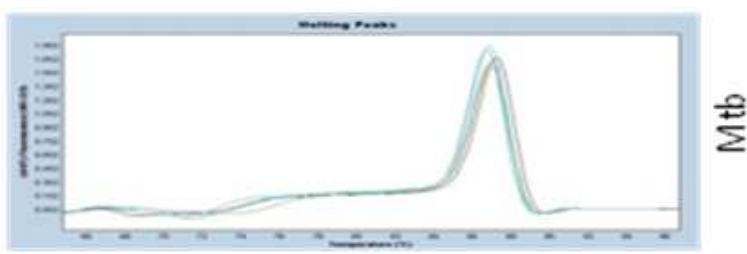

(d)

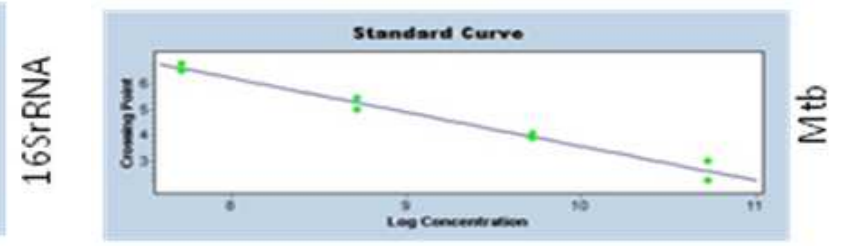

(c)

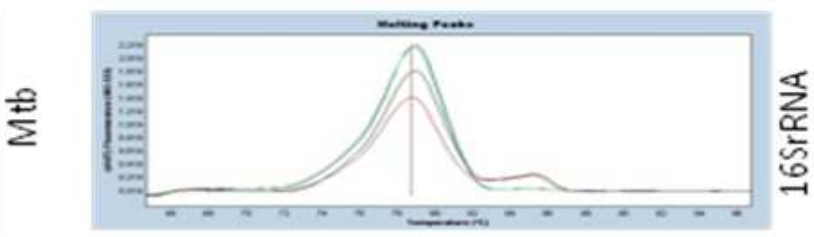

(e)

Fig. 3. FWS microbiome showing polymicrobial infection; (a) Area chart showing dominance of airborne microbes in FWS; (b and c) Standard curves of 16SrRNA and Mtb respectively; (d and e) melting peaks of $M t b$ and 16SrRNA respectively showing amplification of only the specific target sequence 
16SrRNA

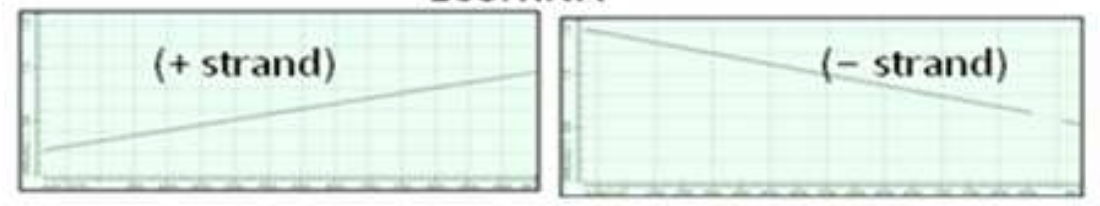

Supplementary Fig. 3. NCBI dot matrix view between the 16SrRNA gene data base (http://greengenes.lbl.gov)
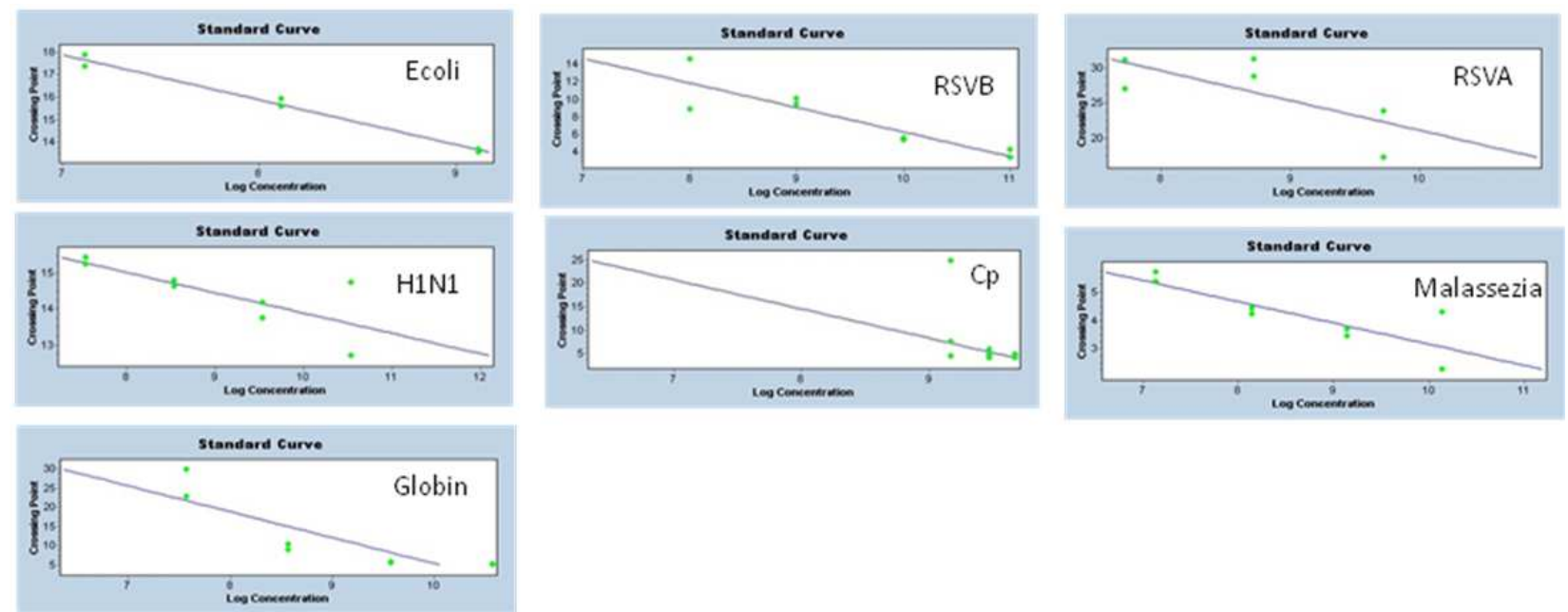

(a)
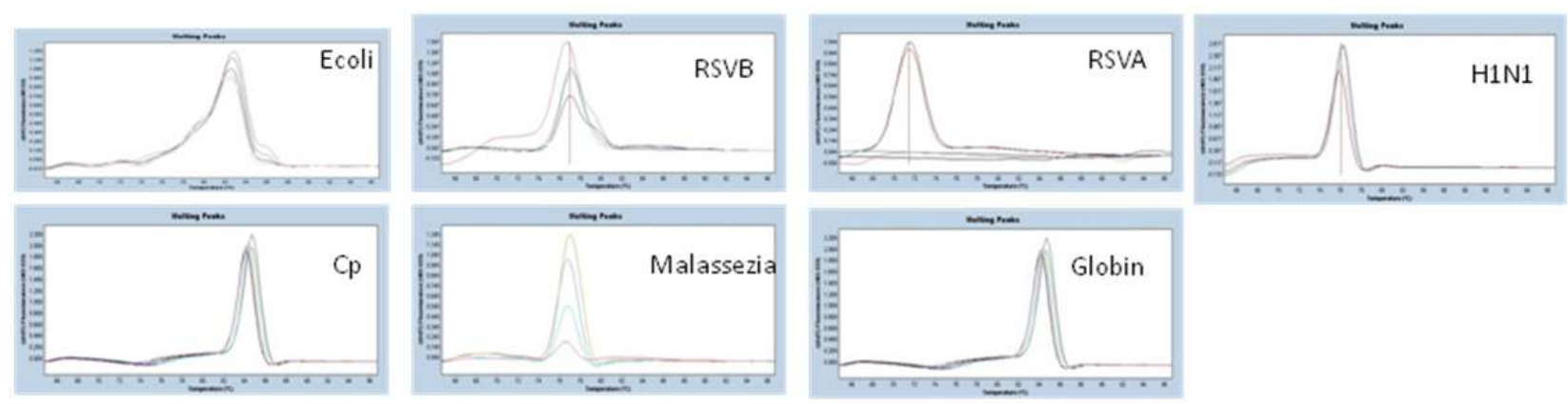

(b)

Supplementary Fig. 4. Standard curves (a) and the first negative derivative of the sample fluorescent curves (b) of different microbes

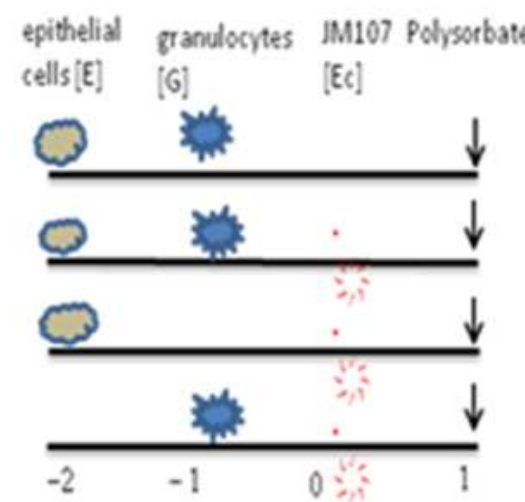

(a)

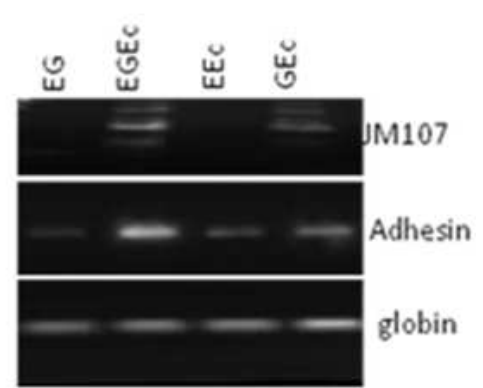

(b) 


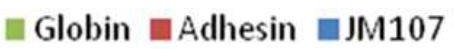

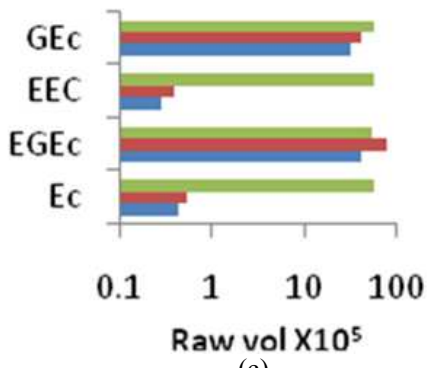

(c)

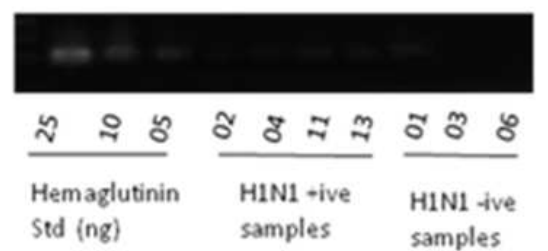

(d)

Fig. 4. Status of binding molecules (Adhesin and H1). E. coli JM107 is unable to simulate adaptation to epithelial cells in absence of granulocytes and lack of adhesin mRNA; (a) protocol scheme; (b) amplicons profiles of E. coli JM107, adhesin and globin from the in vitro skin adaptation/infection assay (EG, epithelial cells + granulocytes; EGEc, epithelial cells + granulocytes + E. coli JM107; EEc, epithelial cells + E. coli JM107; GEc, granulocytes + E. coli JM107). For priming, granulocytes were treated with TNF- $\alpha\left(50 \mathrm{ng} \mathrm{mL}^{-1}\right)$ in vitro for $4 \mathrm{~h}$; (c), densitometric analysis of (b and d) H1 mRNA profiles in flu virus positive and negative FWS samples and varying concentration of Mexican H1N1 lysates (25, 10 and 5 ng). Mexican H1N1lysates represent the positive controls

\section{Discussion}

An abundant and diverse collection of bacteria, fungi and viruses inhabits the human skin. These microorganisms vary between individuals and between different sites on the skin. The factors responsible for the unique variability of the skin microbiota are only partly understood, but results suggest that host genetic and environmental influences play a major role. The steady accumulation of data describing the skin microbiota, combined with experiments designed to test the biological functions of surface microbes, has provided new insights into links between human physiology and skin microbiota (Schommer and Gallo, 2013).

In this investigation we observe a measurable quantum of unviable respiratory and non-respiratory microbes in the scalp epithelium. The clearance of FWS resident microbes benefits the host and in parallel, addresses a critical environmental issue, the local hostmicrobe infection dynamics. The flux of different microbes in FWS can result by direct precipitation from the environment, followed by infection of scalp epidermis, stratum corneum. Both the epithelial cell monolayer and the mucosal surface act as physical and biological barriers against microbial invaders. The integrity of the epithelial monolayer is sustained by tight cell-cell junctions and the mucosal surface is covered by a mucin layer containing various digestive enzymes, Muc2 (a major large gel-forming mucin), secreted IgA and many other antimicrobial agents, including $\beta$ defensins, cathelicidins, bactericidal/permeabilityincreasing protein and chemokines (Leser and Molbak, 2009; Mason and Huffnagle, 2009). Collectively these barriers can prevent infection but possibilities remain that the micro-organisms may breach these barriers and make a foothold on the scalp stratum corneum. We ask, can passively bound airborne microbes adapt to the skin environment and infect through this portal. Adaptation to the skin environment and associated pathogenicity may be due to unique metabolic limitations and capabilities. In case of Malassezia, the fungus is armed with multiple secreted lipases to aid in harvesting host lipids. In addition, an abundance of genes encoding secreted hydrolases (e.g., lipases, phospholipases, aspartyl proteases and acid sphingomyelinases) is found in the Malassezia genome (Xu et al., 2007) that enabled the fungus to adapt to the skin environment and induce pathogenicity. Notably, Mtb possesses numerous lipolytic enzymes belonging to Hormone-Sensitive Lipase (HSL) family, which encompasses twelve serine hydrolases closely related to the human HSL. These enzymes have been characterized and are thought to play critical roles during the intercellular lifetime by participating in the entry into a non-replicating dormant state within host granulomas and/or in dormancy escape, leading to reactivation of the disease (Delorme et al., 2012). Airborne $M t b$ aerosols may utilise these arsenals during entry and colonisation in to the scalp epithelium. On the other hand, the intercellular Mtb bacilli may undergo phagocytosis by antigen-presenting cells (neutrophils, macrophages and dendritic cells) as seen in lung alveoli through the recognition of PathogenAssociated Molecular Patterns (PAMP) by specific pathogen recognition receptors (Akira et al., 2006). It is the key process to initiate and coordinate the host innate immune response. Circulating granulocytes may assist in this process resulting into ARM load in FWS by virtue of phagocytic surveillance against respiratory microbes gaining entry through all susceptible portals like nasal mucosa, conjunctivae and mouth, as well as the scalp or damaged sites on the face. It is possible that infected populations of resident granulocytes at the scalp 
epithelium, as well as infected granulocytes from different loci are transported to inflammed peripheral skin through extravasation for onward shedding along with scalp corneocytes. The latter proposition stems from the presence of $E$. coli infested FWS as $E$. coli is a non-respiratory microbe and primarily infects via fecaloral route. The scattered presence of lymphoid cells and squirting capillaries in the papillary dermis with hints of spongiosis and focal parakeratosis have been shown elsewhere (Piérard-Franchimont et al., 2006). The desquamation of scalp epithelial cells in conjunction with infected granulocytes in response to microbial infection appears pivotal for both the host and environment. Thus, skin shedding plays a significant role in influencing the human pathophysiology as well as the content and character of microbiological aerosols. Non-virulent nature of the FWS, as well as the absence of Adhesin and $\mathrm{H} 1$ is suggestive of minimum risk of re-infection to neighboring cells and subjects. However, other respiratory ailments of humans like allergies and asthma are not ruled out for FWS. This study is likely to help understand the mechanism of innate clearance mechanism of respiratory microbes, as well as the impacts of biological aerosol exposure on human health and well being.

\section{Conclusion}

Desquamated epithelium, in addition to Malassezia $\mathrm{sp}$, bear genomic signatures of diverse non-virulent respiratory microbes, suggest an undefined portal of entry, helps in clearance of microbes and possess minimum risk of re-infection to neighboring cells and subjects. This study will help in understanding the consequences of respiratory infection through noncanonical route(s). Although we have focused on the genomic signatures of ARM in general, identifying dormant variants of antibiotic sensitive microbes in desquamated epithelium will add a new dimension to this investigation. Furthermore, this study encourages comprehensive epidemiological evaluation of genomic signatures on desquamated epithelium in patients with specific infection and control cohorts to help explain the mechanism of transport destination and clearance of infected granulocyte from distant loci. Demonstration of such mechanism may promote developing alternative therapies to treat chronic infections.

\section{Acknowledgement}

We are grateful to Dr. N. Manikcam for sharing reagents, to B.M. Pandey and Anantram Shukla for experimental assistance. We also thank the IITRPublication Committee for review and allocation of manuscript communication registry \# 3260.

\section{Funding Information}

This work was supported by the Council for Scientific and Industrial Research (CSIR) Network Program NWP33 to BNP, University Grant Commission (UGC), New Delhi for fellowship to JKC and Kothari Foundation for the Post doctoral fellowship to VM.

\section{Author's Contributions}

Divyani Paul: Was involved in the experimentation on epithelial cell infection model, acquisition, analysis and interpretation of data.

Jasmeet Kaur Chhabra: Was involved in the experimentation and the acquisition and analysis of data.

Vani Mishra: Was involved in determining copy numbers of microbes and interpretation of data.

Bhola Nath Paul: Obtained funding and was involved in conceptualizing and designing the study, experimentation, acquisition and interpretation of the data, drafting the manuscript and supervising the study overall.

\section{Ethics}

This article is original and contains unpublished material. The corresponding author confirms that all of the other authors have read and approved the manuscript and no ethical issues involved.

\section{References}

Akira, S., S. Uematsu and O. Takeuchi, 2006. Pathogen recognition and innate immunity. Cell, 124: 783-801. DOI: 10.1016/j.cell.2006.02.015

Bartek, I.L., L.K. Woolhiser, A.D. Baughn, R.J. Basaraba and W.R. Jacobs et al., 2014. Mycobacterium tuberculosis Lsr2 is a global transcriptional regulator required for adaptation to changing oxygen levels and virulence. mBio, 5: e01106-14. DOI: $10.1128 / \mathrm{mBio} .01106-14$

Burrows, S.M., W. Elbert, M.G. Lawrence and U. Poschl, 2009. Bacteria in the global atmosphere-part 1: Review and synthesis of literature data for different ecosystems. Atmos. Chem. Phys., 9: 9263-9280. DOI: 10.5194/acp-9-9263-2009

CDC, 2014a. Seasonal Infuenza (Flu). Centre for Disease Control and Prevention.

CDC, 2014b. Respiratory Syncytial Virus Infection (RSV). Centre for Disease Control and Prevention.

Chhabra, J.K., B. Chattopadhyay and B.N. Paul, 2014. SOCS3 dictates the transition of divergent time-phased events in granulocyte TNF- $\alpha$ signaling. Cell. Mol. Immunol., 11: 105-116. DOI: 10.1038/cmi.2013.36

Contini, C., S. Seraceni, R. Cultrera, M. Castellazzi and E. Granieri et al., 2010. Chlamydophila pneumoniae infection and its role in neurological disorders. Interdis. Perspect. Infect. Dis., 2010: 273573-273590. DOI: $10.1155 / 2010 / 273573$ 
Delorme, V., S.V. Diomande, J.F. Cavalier, F. Carriere and L. Kremer et al., 2012. MmPPOX Inhibits Mycobacterium tuberculosis lipolytic enzymes belonging to the hormone-sensitive lipase family and alters mycobacterial growth. PLoS One, 7: e46493-e46493.

DOI: 10.1371/journal.pone.0046493

Croxen, M.A., R.J. Law, R. Scholz, K.M. Keeny and M. Wlodarska et al., 2013. Recent advances in understanding enteric pathogenic Escherichia coli. Clin. Microbiol. Rev., 26: 822-880. PMID: 24092857

Hochreiter-Hufford, A. and K.S. Ravichandran, 2013. Clearing the dead: Apoptotic cell sensing, recognition, engulfment and digestion. Cold Spr. Harb. Perspect. Biol., 5: a008748-a008748. DOI: 10.1101/cshperspect.a008748

Kim, M., H. Ashida, M. Ogawa, Y. Yoshikawa and H. Mimuro et al., 2010. Bacterial interactions with the host epithelium. Cell Host Microbe, 8: 20-35. DOI: 10.1016/j.chom.2010.06.006

Kim, O.S., Y.J. Cho, K. Lee, S.H. Yoon and M. Kim et al., 2012. Introducing EzTaxon-e: A prokaryotic $16 \mathrm{~S}$ rRNA gene sequence database with phylotypes that represent uncultured species. Int. J. Syst. Evolut. Microbiol., 62: 716-721. DOI: 10.1099/ijs.0.038075-0

Clements, A., J.C. Young, N. Constantinou and G. Frankel, 2012. Infection strategies of enteric pathogenic Escherichia coli. Gut Microbes, 3: 71-78. DOI: $10.4161 /$ gmic. 19182

Leser, T.D. and L. Mølbak, 2009. Better living through microbial action: The benefits of the mammalian gastrointestinal microbiota on the host. Environ. Microbiol., 11: 2194-2206.

DOI: $10.1111 / \mathrm{j} .1462-2920.2009 .01941 . x$

Mason, K.L. and G.B. Huffnagle, 2009. Control of mucosal polymicrobial populations by innate immunity. Cell. Microbiol., 11: 1297-1305. DOI: $10.1111 / \mathrm{j} .1462-5822.2009 .01347 . \mathrm{x}$

Miller, M.J., 2013. Two-photon imaging of immune cell dynamics: Moving from 2D fixed tissue sections to 3D dynamic histology in vivo. Blood.

Piérard-Franchimont, C., E. Xhauflaire-Uhoda and G.E. Piérard, 2006. Revisiting dandruff. Int. J. Cosmet. Sci., 28: 311-318. DOI: $10.1111 / \mathrm{j} .1467-2494.2006 .00326 . x$

Saunders, C.W., Scheynius, A. and J. Heitman, 2012. Malassezia Fungi are specialized to live on skin and associated with dandruff, Eczema and other skin Diseases. PLoS Pathog., 8: e1002701-e1002701. DOI: $10.1371 /$ journal.ppat.1002701
Schommer, N.N. and R.L. Gallo, 2013. Structure and function of the human skin microbiome. Trends Microbiol., 21: 660-668. DOI: $10.1016 /$ j.tim.2013.10.001

Shelton, H., G. Ayora-Talavera, J. Ren, S. Loureiro and R.J. Pickles et al., 2011. Receptor Binding profiles of Avian Influenza virus hemagglutinin subtypes on human cells as a predictor of pandemic potential. J. Virol., 85: 1875-1880. DOI: 10.1128/JVI.01822-10

Weninger, W., M. Biro and R. Jain, 2014. Leukocyte migration in the interstitial space of non-lymphoid organs. Nat. Rev. Immunol., 14: 232-246.

DOI: $10.1038 /$ nri3641

Whon, T.W., M.S. Kim, S.W. Roh, N.R. Shin and H.W. Lee et al., 2012. Metagenomic characterization of airborne Viral DNA diversity in the near-surface atmosphere. J. Virol., 86: 8221-8231.

DOI: $10.1128 /$ JVI.00293-12

Woo, P.C.Y., S.K.P. Lau, J.L.L. Teng, H. Tse and K.Y., Yuen, 2008. Then and now: Use of 16S rDNA gene sequencing for bacterial identification and discovery of novel bacteria in clinical microbiology laboratories. Clin. Microbiol. Infect., 14: 908-934. DOI: $10.1111 /$ j.1469-0691.2008.02070.x

WHO, 2014. Tuberculosis fact sheet $\mathrm{N}^{\circ} 104$. World Health Organization.

Wu, C.F., J.J. Valdes, W.E. Bentley and J.W. Sekowski, 2003. DNA microarray for discrimination between pathogenic 0157:H7 EDL933 and non-pathogenic Escherichia coli strains. Biosens. Bioelecton., 19: 1-8. DOI: 10.1016/S0956-5663(03)00118-0

$\mathrm{Xu}$, J., C.W. Saunders, P. Hu, R.A. Grant and T. Boekhout et al., 2007. Dandruff-associated Malassezia genomes reveal convergent and divergent virulence traits shared with plant and human fungal pathogens. Proc. Nat. Acad. Sci. USA, 104: 18730-18735.

DOI: $10.1073 /$ pnas.0706756104

Zemanick, E.T., B.D. Wagner, S.D. Sagel, M.J. Stevens and F.J. Accurso et al., 2010. Reliability of quantitative real-time PCR for bacterial detection in cystic fibrosis airway specimens. PLoS ONE, 5: e15101-e15101. DOI: 10.1371/journal.pone.0015101 